\title{
Redes metalorgânicas à base de zinco e alumínio como catalisadores heterogêneos para a esterificação do ácido oleico
}

\author{
Metal-Organic Frameworks based in zinc and \\ aluminium as heterogeneous catalysts to \\ oleic acid esterification
}

Lorena Costa Alves ${ }^{1}$, Fernanda Gabriel de Freitas ${ }^{1}$, Fernando Cesário Rangel ${ }^{1}$, Rosenira Serpa da Cruz ${ }^{1}$

\footnotetext{
${ }^{1}$ Grupo Bioenergia e Meio Ambiente, BIOMA/UESC, Departamento de Ciências Exatas e Tecnológicas, Universidade Estadual de Santa Cruz, Campus Soane Nazaré de Andrade, Rodovia Jorge Amado, km 16, Salobrinho, Ilhéus, Bahia, Brasil.

e-mail: lorenaalves_net@hotmail.com, fergfreitas@hotmail.com, fcrangel@uesc.br; roserpa@uesc.br
}

\section{RESUMO}

Os problemas ambientais recorrentes do uso de combustíveis fósseis incentivaram a busca por combustíveis de fontes limpas e renováveis, capazes de minimizar a emissão de gases que causam o efeito estufa. Neste contexto, o uso do biodiesel vem se destacando no mercado de combustíveis. A produção industrial de biodiesel baseada na catálise alcalina homogênea apresenta exigências técnicas que impactam diretamente no seu custo de produção. Pesquisadores têm buscado desenvolver catalisadores heterogêneos, como uma alternativa a este processo. Rede metalorgânicas, MOFs é uma classe de materiais promissores para processos catalíticos, uma vez que resultam da combinação de materiais orgânicos e inorgânicos, o que lhes confere propriedades como alta porosidade, estrutura ordenada e flexibilidade estrutural. Desta forma, o enfoque deste estudo foi sintetizar e caracterizar MOFs, contendo óxidos de zinco e alumínio preparados através do método hidrotérmico, e imobilizá-los na rede de sílica, visando um aumento da sua estabilidade térmica e química. As caracterizações físico-químicas dos materiais confirmaram a formação de redes metalorgânicas, indicando que o método de síntese foi reprodutível e eficiente. Os testes catalíticos indicaram que o material não imobilizado MOF-Al/Zn apresentou maior atividade catalítica na esterificação do ácido oleico, no entanto apresentou lixiviação das espécies ativas. Contudo, a imobilização deste material em sílica resultou em um catalisador estável, sendo possível reutilizá-lo sem perda significativa da atividade catalítica. A imobilização de redes metalorgânicas em sílica se mostrou uma opção promissora para a síntese de um catalisador heterogêneo ativo e eficiente para reações de esterificação do ácido oleico.

Palavras-chave: Biodiesel, redes metalorgânicas, sílica, reações de esterificação.

\section{ABSTRACT}

The recurring environmental problems of the use of fossil fuels have encouraged the search for fuels from clean and renewable sources that can minimize the emission of greenhouse gases. In this sense, biodiesel has been highlighted in the market of fuels. The production of industrial biodiesel based on homogeneous alkaline catalysis presents technical requirements that directly impact on its cost of production. Researchers have sought to develop heterogeneous catalysts as an alternative to this process. Metal-Organic Frameworks, MOFs, are a class of promising materials for catalytic processes, as they result from the combination of organic and inorganic materials, which gives them properties such as high porosity, ordered structure and structural flexibility. Thus, the focus of this study was to synthesize and characterize MOFs containing zinc and aluminum oxides, prepared by the hydrothermal method, and immobilize them in the silica network, aiming to increase their thermal and chemical stability. The physicochemical characterization of the materials confirmed the formation of MOFs, indicating that the synthesis method was reproducible and efficient. The catalytic tests indicated that the non-immobilized material MOF-Al/Zn presented higher catalytic activity in the 
esterification of oleic acid, however it showed leaching of the active species. However, the immobilization of this material on silica resulted in a stable catalyst, being possible to reuse it without significant loss of the catalytic activity. The immobilization of Metal-Organic Frameworks on silica networks has shown to be a promising option for the synthesis of an active and efficient heterogeneous catalyst for esterification reactions of oleic acid.

Keywords: Biodiesel, metal-organic frameworks, silica, esterification reactions.

\section{INTRODUÇÃO}

O crescimento econômico mundial e o consumo exagerado dos combustíveis fósseis nos últimos anos impulsionaram o desenvolvimento de pesquisas, na busca por fontes alternativas de energia que possam diminuir os impactos ambientais e problemas que envolvem questões geopolíticas [1,2]. Pesquisadores investem seu tempo no desenvolvimento de novas tecnologias que possam minimizar a dependência desses combustíveis de origem fóssil, reduzir a emissão de gases poluentes e os problemas de âmbito social e econômico [1-3].

Dentre os recursos renováveis com inovações tecnológicas em desenvolvimento e com utilização em escala crescente destaca-se a biomassa, a qual inclui qualquer matéria orgânica que se encontra disponível em uma base renovável e sua conversão é capaz de gerar uma grande variedade de produtos químicos com alto valor agregado [4]. O biodiesel, proveniente da biomassa vem se destacando no mercado devido a sua natureza menos poluente e renovável. A evolução na sua produção está diretamente ligada às vantagens ambientais que ele apresenta em relação aos combustíveis fósseis, como além do seu caráter renovável, o aumento da eficiência da combustão [5], emissões mais limpas [5,6], alto número de cetano, alto ponto de fulgor, melhor lubrificação e biodegradabilidade [6,7].

O método mais utilizado na produção industrial de biodiesel é a reação de transesterificação alcalina homogênea. Porém, para que essa rota de produção seja econômica e tecnicamente viável é necessária à utilização de óleo com baixa porcentagem de ácido graxo livre e umidade, além de necessitar de etapas consecutivas de lavagem para separação e purificação do produto, neutralização do catalisador e recuperação da glicerina [6]. Por isso, uma incessante busca por processos que utilizem catalisadores heterogêneos eficientes em condições reacionais brandas, para que o biodiesel se torne mais competitivo do ponto de vista de aplicação industrial $[7,8]$.

A produção de biodiesel via catálise heterogênea se apresenta como uma alternativa ecologicamente correta, do ponto de vista de geração de efluentes, pois, não favorecem as reações de saponificação, dispensa a etapa de purificação do produto, já que sua ação ocorre em fase diferente do meio reacional o que possibilita uma simplificação na separação do catalisador e do produto, este catalisador separado pode ser reutilizado nos próximos processos, resultando em benefícios ambientais, técnicos e econômicos [8,9].

Sólidos cristalinos porosos têm atraído continuamente o interesse na comunidade científica devido à sua aplicação em diversos processos tecnológicos [10]. O desenvolvimento de zeólitas foi uma revolução na química do estado sólido, e aluminossilicatos cristalinos porosos são encontrados em numerosas aplicações na indústria atualmente. Mais recentemente, outra classe de materiais chamou a atenção de cientistas das universidades e indústrias: redes metalorgânicas (MOFs), também chamados de polímeros de coordenação porosos. Estes são constituídos por moléculas orgânicas multi-funcionalizadas que são ligadas por unidades inorgânicas para formar sólidos porosos com uma geometria regular e muitas vezes previsíveis [11]. A síntese, caracterização e aplicação da MOF em campos de grande interesse tecnológico são devido à suas propriedades únicas, e, como resultado, houve um aumento exponencial do número de publicações observadas nas últimas décadas, já que a química e sua versatilidade estrutural tornam esses materiais grandes candidatos para novas aplicações [11-13].

Uma grande variedade de MOFs já foi sintetizada com vários metais de transição, bem como com diferentes ligantes e se destacaram como catalisadores heterogêneos em diferentes transformações orgânicas [13-16]. Dentro desta premissa, o uso de MOFs como catalisadores heterogêneos para a produção de biodiesel destaca-se como uma opção promissora, uma vez que apresentam tamanhos de poros favoráveis para a difusão de substratos, tais como os ésteres [17-21]. Além disso, permite alcançar uma distribuição homogênea de um ou mais sítios ativos, devido ao seu grau de cristalinidade, possibilita adicionar funcionalidades durante ou pós-síntese, de modo a ajustar não só a força ácido-base, mas também, o caráter hidrofílicohidrofóbico da superfície, além de apresentarem altas áreas superficiais específicas [12,22,23]. Diante do exposto, este trabalho teve como objetivo sintetizar MOFs contendo óxidos dos metais Al/Zn através do método hidrotérmico e imobilizar na matriz de sílica, a fim de se obter um material com caráter ácido, mais estável térmica e quimicamente e que possa ser aplicado como um catalisador heterogêneo em reação de esterificação de ácido graxo com vistas à aplicação na indústria de biodiesel. 


\section{MATERIAIS E MÉTODOS}

Síntese das redes metalorgânicas à base de alumínio e zinco:

Em $300 \mathrm{~mL}$ de dimetilformamida (DMF- $\left.\mathrm{C}_{3} \mathrm{H}_{7} \mathrm{NO}\right)$ foram adicionados $16,6 \mathrm{~g}$ de ácido tereftálico $\left(\mathrm{C}_{8} \mathrm{H}_{6} \mathrm{O}_{4}-0,1\right.$ mol L ${ }^{-1}$ ), sendo que a mistura reacional formada foi aquecida sob constante agitação. Após a dissolução completa do ácido, foram adicionadas a mistura reacional 5,6 $\mathrm{g}$ de $\mathrm{Al}\left(\mathrm{NO}_{3}\right)_{3} \cdot 9 \mathrm{H}_{2} \mathrm{O}$ e $4,5 \mathrm{~g}$ de $\mathrm{Zn}\left(\mathrm{NO}_{3}\right)_{2} \cdot 6 \mathrm{H}_{2} \mathrm{O}$, ambos contento concentrações de $0,1 \mathrm{~mol} \mathrm{~L}^{-1}$, sendo o sistema reacional selado e mantido sob refluxo por 17 h. Após o resfriamento da solução até à temperatura ambiente, o produto foi centrifugado e lavado duas vezes com DMF e cinco vezes com etanol, até a sua purificação. O produto obtido foi recolhido e seco a $105^{\circ} \mathrm{C}$ por um período de $4 \mathrm{~h}$ e em temperatura de $150^{\circ} \mathrm{C}$ sob vácuo durante um tempo de $6 \mathrm{~h}$. A amostra obtida sob a forma de pó foi designada com a sigla MOF-Al/Zn [24].

\subsection{Imobilização da MOF-Al/Zn em sílica}

Nessa etapa a sílica foi preparada pelo método sol-gel na presença da MOF-Al/Zn. Em resumo, foram adicionados 115,0 g de etanol, $52 \mathrm{~mL}$ de tetraetoxisilano (TEOS), $40 \mathrm{~mL}$ de $\mathrm{NH}_{4} \mathrm{OH} 0,005 \mathrm{~mol} \mathrm{~L}^{-1}$ e 3,0 g da MOF-Al/Zn sob agitação, em banho-maria a uma temperatura de $70^{\circ} \mathrm{C}$ até completa homogeneização. Esta mistura permaneceu em repouso, sob temperatura ambiente por 5 dias, quando ocorreu a gelatinização. Em seguida o material foi seco em estufa a $100^{\circ} \mathrm{C}$ por um período de $3 \mathrm{~h}$. Este material foi denominado MOF$\mathrm{Al} / \mathrm{Zn}-\mathrm{SiO}_{2}$.

\subsection{Caracterização físico-química dos materiais}

Os espectros na região do infravermelho foram coletados em um espectrofotômetro da marca ThermoScientificicolet Modelo SI10, com faixa de 4000 a $400 \mathrm{~cm}^{-1}$ e uma resolução de $4 \mathrm{~cm}^{-1}$. As amostras caracterizadas foram preparadas na forma de pastilha de $\mathrm{KBr}$ com uma proporção de $1 \%$ dos sólidos.

Os padrões de raios X foram obtidos em um Difratômetro da Rigaku, modelo Miniflex 600, empregando radiação $\mathrm{Cu}-\kappa \alpha$, operando a $40 \mathrm{kV}$ e $15 \mathrm{~mA}$. A velocidade do goniômetro foi de $10^{\circ} \mathrm{C} \cdot \mathrm{min}^{-1} \mathrm{e}$ as faixas de difração em $2 \theta$ vão de $10^{\circ}$ a $100^{\circ}$.

As imagens de microscopia eletrônica de varredura foram realizadas em um microscópio eletrônico de varredura, marca Quanta 250 , de baixo vácuo e tensão de $30 \mathrm{kV}$.

As isotermas de adsorção-dessorção de nitrogênio foram obtidas a 77K usando um aparelho Micrometrics ASAP 2420. A amostra foi previamente tratada a $450^{\circ} \mathrm{C}$, em seguida submetida a fluxo de nitrogênio, por $14 \mathrm{~h}$.

As análises termogravimétricas foram analisadas em equipamento da Schimadzu DTG - 60/60H, onde as amostras sólidas foram aquecidas a taxa de $10^{\circ} \mathrm{C} \mathrm{min}^{-1}$, de $25^{\circ} \mathrm{C}$ até $800^{\circ} \mathrm{C}$, em fluxo de nitrogênio a 50 $\mathrm{mL} \cdot \mathrm{min}^{-1}$, empregando porta amostra de alumina.

\subsection{Avaliação catalítica dos materiais}

Os materiais foram avaliados na reação de esterificação do ácido oleico com metanol, empregando as seguintes condições reacionais: razão metanol (SIGMA ALDRICH, 98\%): ácido oleico (SIGMA ALDRICH, 99\%) $6: 1,5 \% \mathrm{~m} / \mathrm{m}$ de catalisador em relação ao ácido oleico, $3 \mathrm{~h}$ de reação e temperatura de $150{ }^{\circ} \mathrm{C}$ em um reator Parr de $300 \mathrm{~mL}$. Antes do início da reação, os materiais foram ativados a $120^{\circ} \mathrm{C}$ por $1 \mathrm{~h}$.

Para verificar a ocorrência ou não de lixiviação das espécies ativas para o meio reacional, seguiu-se uma metodologia segundo Ulrich et al. [25], onde foi preparada uma reação padrão e após $1 \mathrm{~h}$, filtrou-se a solução a quente, para a retirada do catalisador e à porção filtrada foi retomada para a reação. Este sistema foi colocado sob as mesmas condições reacionais por mais 6 horas e, então foram retiradas alíquotas a cada $1 \mathrm{~h}$ para acompanhar o andamento da reação até a sua finalização.

Os materiais foram avaliados quanto à capacidade de reutilização, onde as reações seguiram as mesmas condições descritas para as reações de esterificação, sendo que após um ciclo reacional de $3 \mathrm{~h}$, foi efetuada a filtração do material seguida de lavagem com metanol. O catalisador foi seco em estufa a $120^{\circ} \mathrm{C}$ por $1 \mathrm{~h}$, pesado e reutilizado em nova reação, mantendo-se sempre as mesmas condições reacionais. Tal procedimento foi realizado para quatro ciclos. 


\section{RESULTADOS E DISCUSSÃO}

\subsection{Caracterizações físico-química dos materiais}

Através das análises dos espectros de absorção na região do infravermelho foram identificados os grupos funcionais presentes nas redes metalorgânicas sintetizadas pelo método do ácido tereftálico. Os espectros de infravermelho dispostos na Figura 1, representam as amostras de ácido tereftálico puro, MOF-Al/Zn, MOF$\mathrm{Al} / \mathrm{Zn}-\mathrm{SiO}_{2}$. Os espectros apresentam absorções nas regiões de $1600-1500 \mathrm{~cm}^{-1}$ que correspondem às vibrações de $\mathrm{COO}^{-}$simétrico e assimétrico, respectivamente, bandas em torno de $1400 \mathrm{~cm}^{-1}$ correspondem às vibrações das ligações $\mathrm{C}=\mathrm{C}$ de aromático. As faixas de absorções entre $950 \mathrm{~cm}^{-1}, 800 \mathrm{~cm}^{-1} \mathrm{e} 650 \mathrm{~cm}^{-1}$ podem ser atribuídas às vibrações de estiramento no plano e fora do plano de ligações $\mathrm{C}-\mathrm{H}$ do anel do benzeno presente na estrutura do ligante que se mantém após a coordenação com metais zinco e alumínio. As bandas entre 3200 e $3400 \mathrm{~cm}^{-1}$ representam as moléculas de água que estão coordenadas sobre as estruturas formadas. Os modos vibracionais dos grupos silanóis foram identificados pelas bandas nas regiões de 1084, 970, 789 e $463 \mathrm{~cm}^{-1}$, confirmando a formação da matriz inorgânica [12,23].

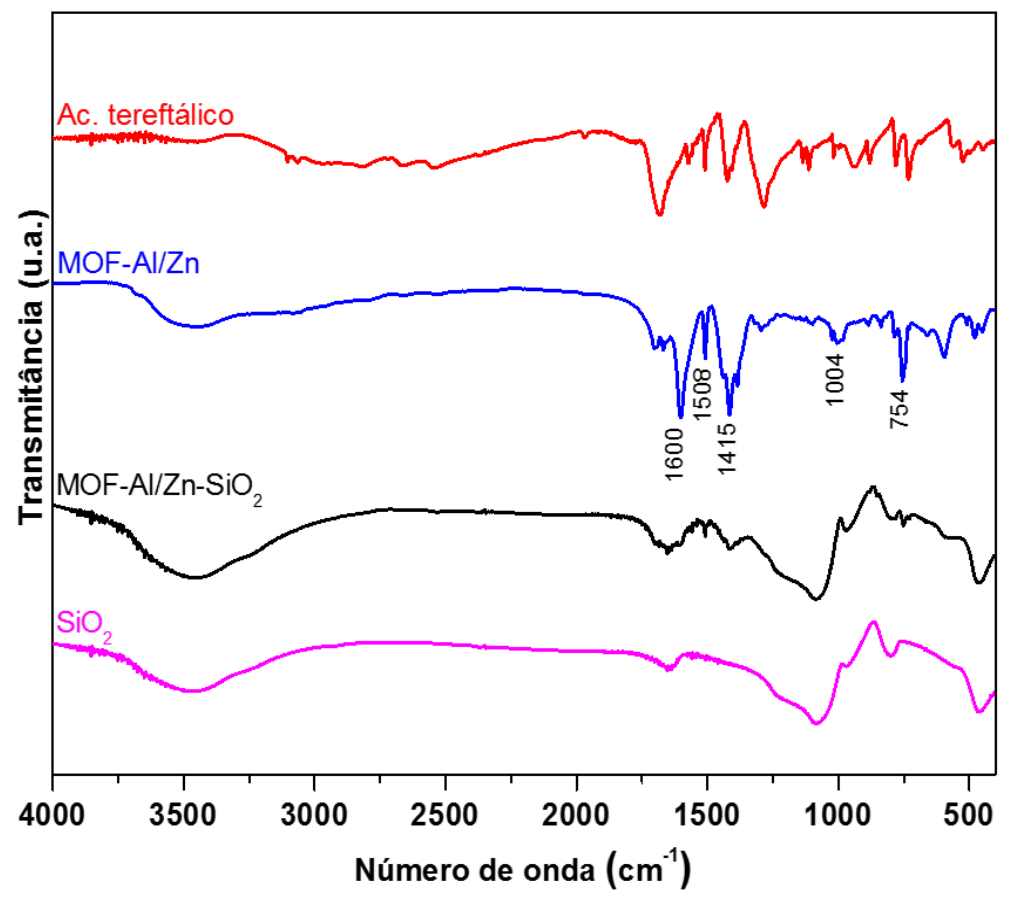

Figura 1: Espectros de absorção na região do infravermelho das amostras do ligante ácido tereftálico, MOF-Al/Zn, $\mathrm{MOF}-\mathrm{Al} / \mathrm{Zn}-\mathrm{SiO}_{2}$ e da sílica pura.

Os padrões de difração de raios X, Figura 2, para a amostra MOF-Al/Zn, confirmou a formação de uma rede metalorgânica organizada [24], uma vez que o material apresentou pico intenso em baixos ângulos na faixa de $10^{\circ}$, caracterizando redes metalorgânicas sintetizadas por processos do tipo hidrotérmico [13,18,22,24,26,27]. O material imobilizado na rede de sílica apresentou picos mais largos evidenciando o caráter amorfo do compósito sintetizado e a boa dispersão do MOF-Al/Zn sobre a matriz, como já indicado na literatura por outros autores [13,14,24,26,27].

As análises termogravimétricas das MOFs sintetizadas pelo método hidrotérmico estão representadas na Figura 3. As análises dos materiais indicaram que tanto a MOF-Al/Zn quanto a MOF-Al/Zn- $\mathrm{SiO}_{2}$ apresentaram boa estabilidade térmica. A degradação térmica do material MOF-Al/Zn começou em temperaturas próximas a $490^{\circ} \mathrm{C}[24,27]$ antes disso ocorreram os eventos descritos como: a primeira etapa foi identificada pela evaporação de moléculas de água coordenadas a estrutura ou do solvente dimetilformamida, que foram representadas na faixa de temperatura entre $100^{\circ} \mathrm{C}$ e $200^{\circ} \mathrm{C}$, já a segunda etapa ocorreu devido à eliminação de moléculas de DMF que estavam coordenadas a estrutura do material, que ocorre em temperaturas entre $200^{\circ} \mathrm{C}$ e $400^{\circ} \mathrm{C}$ e em temperaturas superiores a $500^{\circ} \mathrm{C}$, ocorreu o processo de degradação da amostra 
$[20,24,27]$. Para o material suportado, $\mathrm{MOF}-\mathrm{Al} / \mathrm{Zn}-\mathrm{SiO}_{2}(\mathrm{O})$, houve uma perda de massa na faixa de $100^{\circ} \mathrm{C}$ e outra pequena perda de massa na temperatura de $490^{\circ} \mathrm{C}$ que corresponde a saída de moléculas de DMF coordenadas sobre a estrutura da MOF, porém a curva de termogravimetria do material suportado se manteve estável até atingir a temperatura de $800^{\circ} \mathrm{C}$, indicando que o material suportado na rede de sílica apresentou maior estabilidade quando comparada ao mesmo material não suportado, demonstrando que a incorporação da sílica proporcionou ao material um aumento em sua estabilidade térmica.

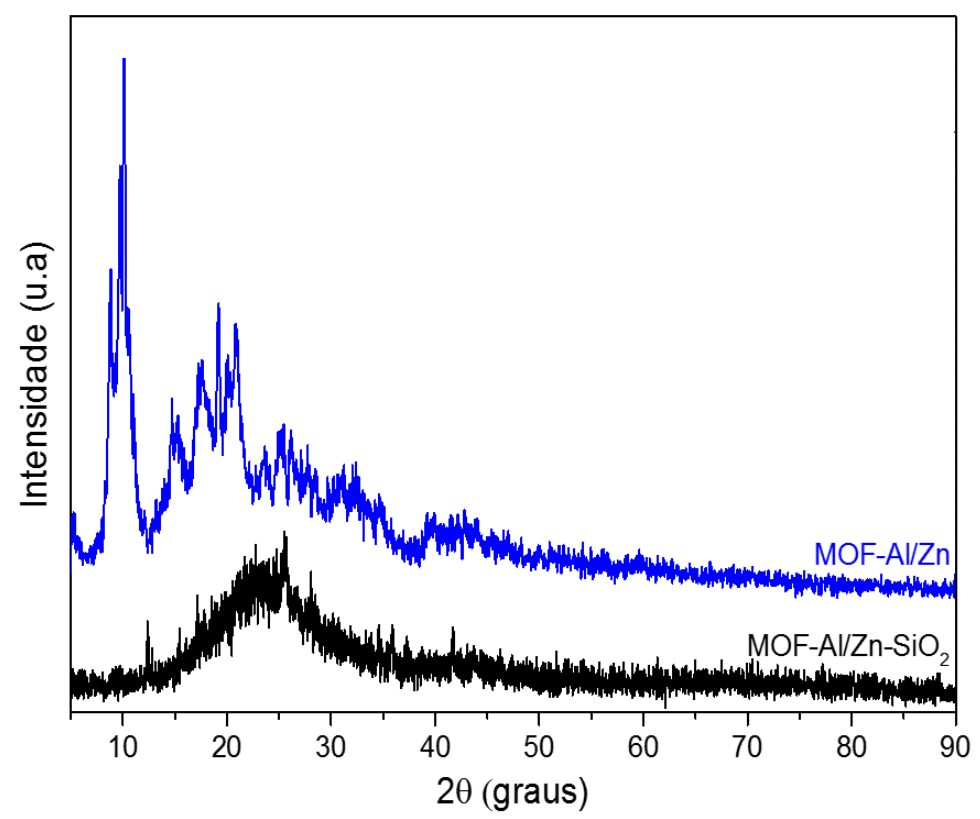

Figura 2: Padrões de de raios $\mathrm{X}$ dos materiais MOF-Al/Zn e MOF-Al/Zn-SiO ${ }_{2}$

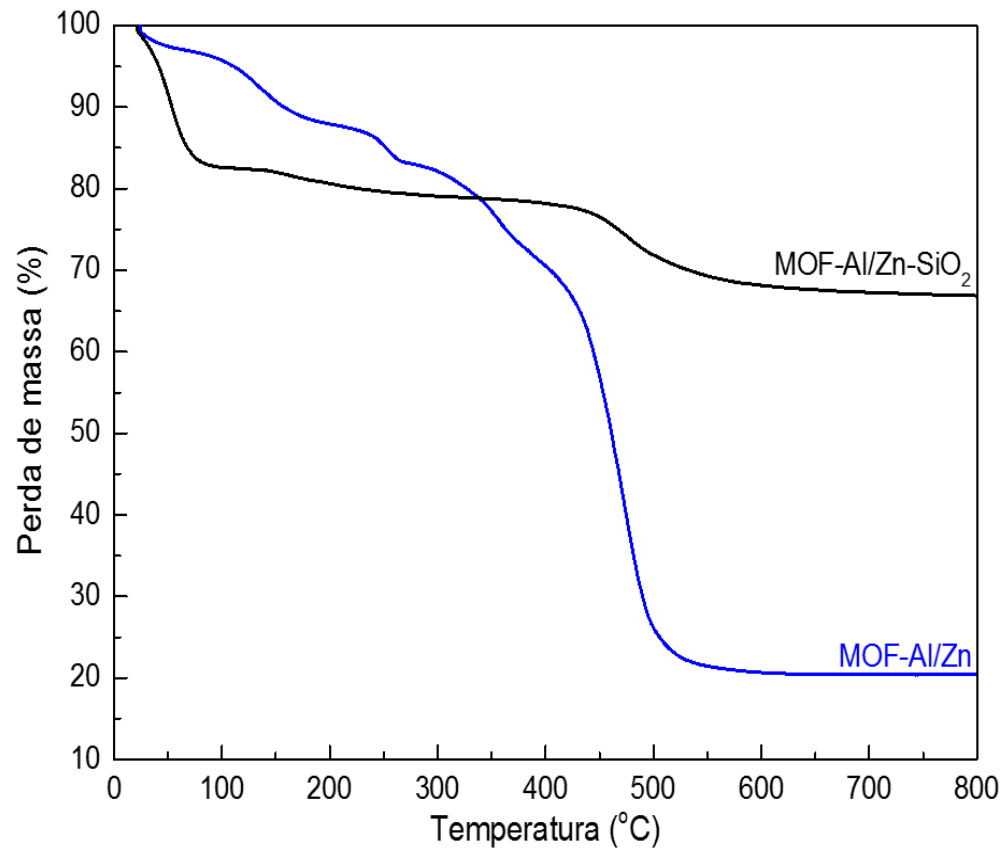

Figura 3: Análises termogravimétricas das redes metalogânicas sintetizadas pelo método hidrotérmico.

A Figura 4 ilustra as imagens de microscopia eletrônica de varreduras (MEV) obtidas para as amostras em estudo, essa técnica permitiu analisar a morfologia, textura e o tamanho dos cristais obtidos para os compostos. 

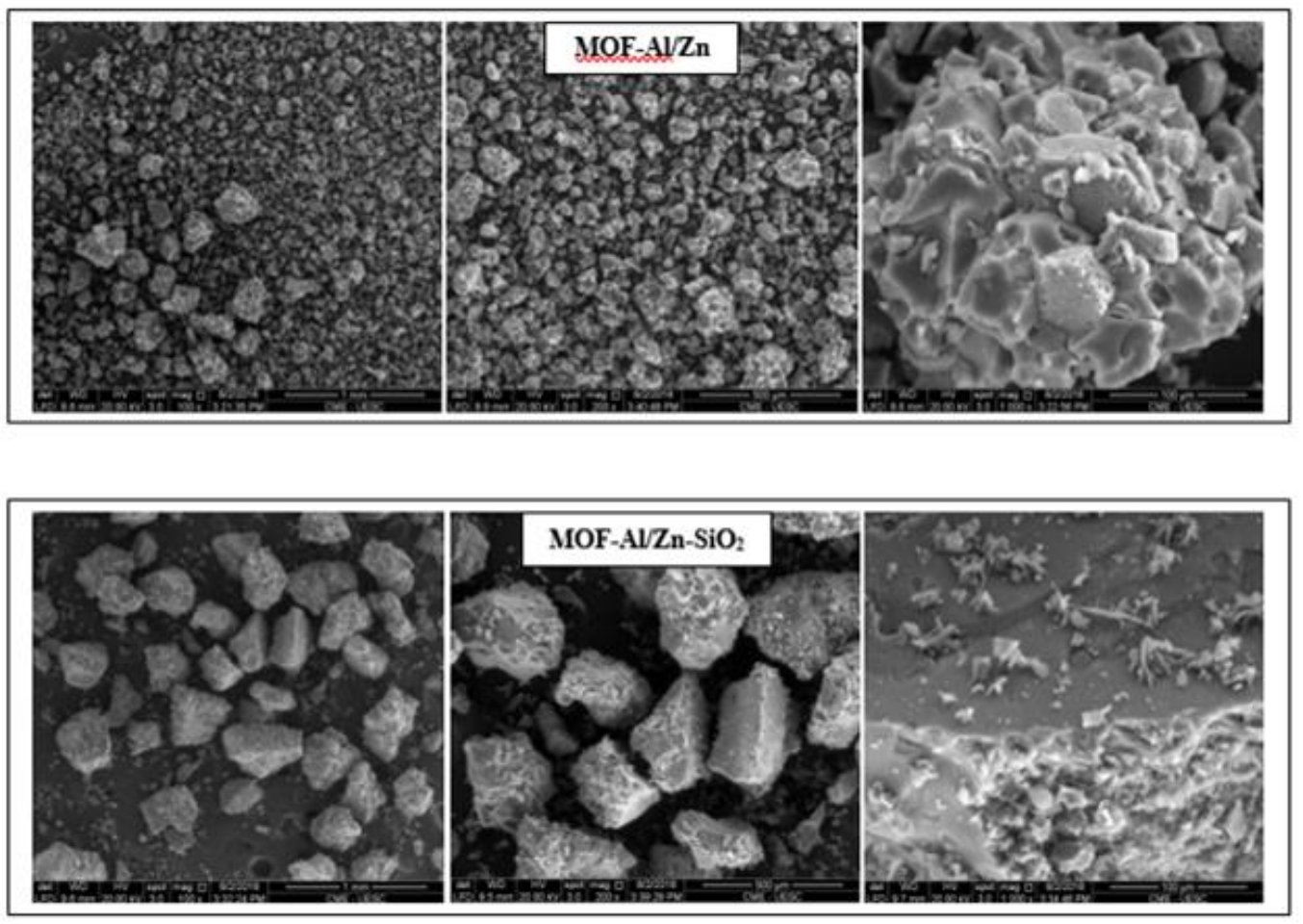

Figura 4: Imagens de MEV das amostras MOF-Al/Zn e MOF-Al/Zn- $\mathrm{SiO}_{2}$.

As imagens indicam que o material MOF-A1/Zn apresentou partículas com tamanhos variados, tamanhos de poros inferior a $10 \mu \mathrm{m}$ e sua geometria se assemelha a um octaedro. O material MOF-Al/Zn-SiO ${ }_{2}$ apresentou partículas com tamanhos mais uniformes, porém com geometrias irregulares, este aspecto está vinculado à imobilização da MOF-Al/Zn na rede de sílica, corroborando com as análises de DRX e FTIR já discutidas anteriormente.

A técnica de adsorção e dessorção de $\mathrm{N}_{2}$ mostrou que material MOF-Al/Zn apresentou um valor de área específica de $267 \mathrm{~m}^{2} \mathrm{~g}^{-1}$ e diâmetro médio de poros na faixa de 8,2 nm. A imobilização do MOF-Al/Zn na matriz sílica resultou em um material com área especifica de $346 \mathrm{~m}^{2} \mathrm{~g}^{-1}$ e valor de diâmetro médio de poros de 2,4 nm. Estes resultados concordam com as técnicas de caracterização apresentadas, confirmando a formação do compósito da MOF com a sílica com boa dispersão da MOF na matriz, como já indicado e discutido em trabalhos anteriores [26,27].

\subsection{Avaliação catalítica dos materiais}

Os materiais sintetizados foram submetidos à avaliação catalítica por esterificação do ácido oleico. O ácido oleico foi utilizado como molécula modelo na avaliação catalítica e os catalisadores se mostraram ativos para esta reação. Os centros metálicos das redes metalorgânicas atuam como sítios ácidos de Lewis que favorece o emprego destes em reações de esterificação, onde as moléculas de ácido graxo são adsorvidas na superfície do catalisador devido à interação ácido-base entre o par de elétrons do oxigênio carbonílico do ácido graxo e o metal presente na estrutura do catalisador $[4,12,13]$. 
A MOF-Al/Zn se mostrou ativa frente a reação de esterificação com conversão do ácido oleico de $48,4 \pm 1,6 \%$. No entanto, este material solubilizou no meio reacional como comprovado pelo teste de lixiviação, que mostrou que após $2 \mathrm{~h}$ da retirada do catalisador por filtração a quente, a conversão do ácido oleico atingiu o valor de 46,2 $\pm 1,9 \%$ [13]. O material imobilizado com sílica, $\mathrm{MOF}-\mathrm{Al} / \mathrm{Zn}-\mathrm{SiO}_{2}$, apresentou uma conversão em ésteres metílicos de $39,0 \pm 1,3 \%$ após $3 \mathrm{~h}$ e de $59,8 \pm 1,6 \%$ após 6 h de reação e se mostrou estável ao teste de lixiviação, demonstrando que o MOF-Al/Zn ao ser imobilizado na rede de sílica, aumentou as forças de ligação do material final formado [13,23]. O material MOF-Al/Zn-SiO $\mathrm{S}_{2}$ foi então, submetido ao experimento de reutilização, e após quatro ciclos consecutivos não apresentou perda significativa de atividade catalítica. A Figura 5 mostra os resultados destas avaliações (Figura 5).

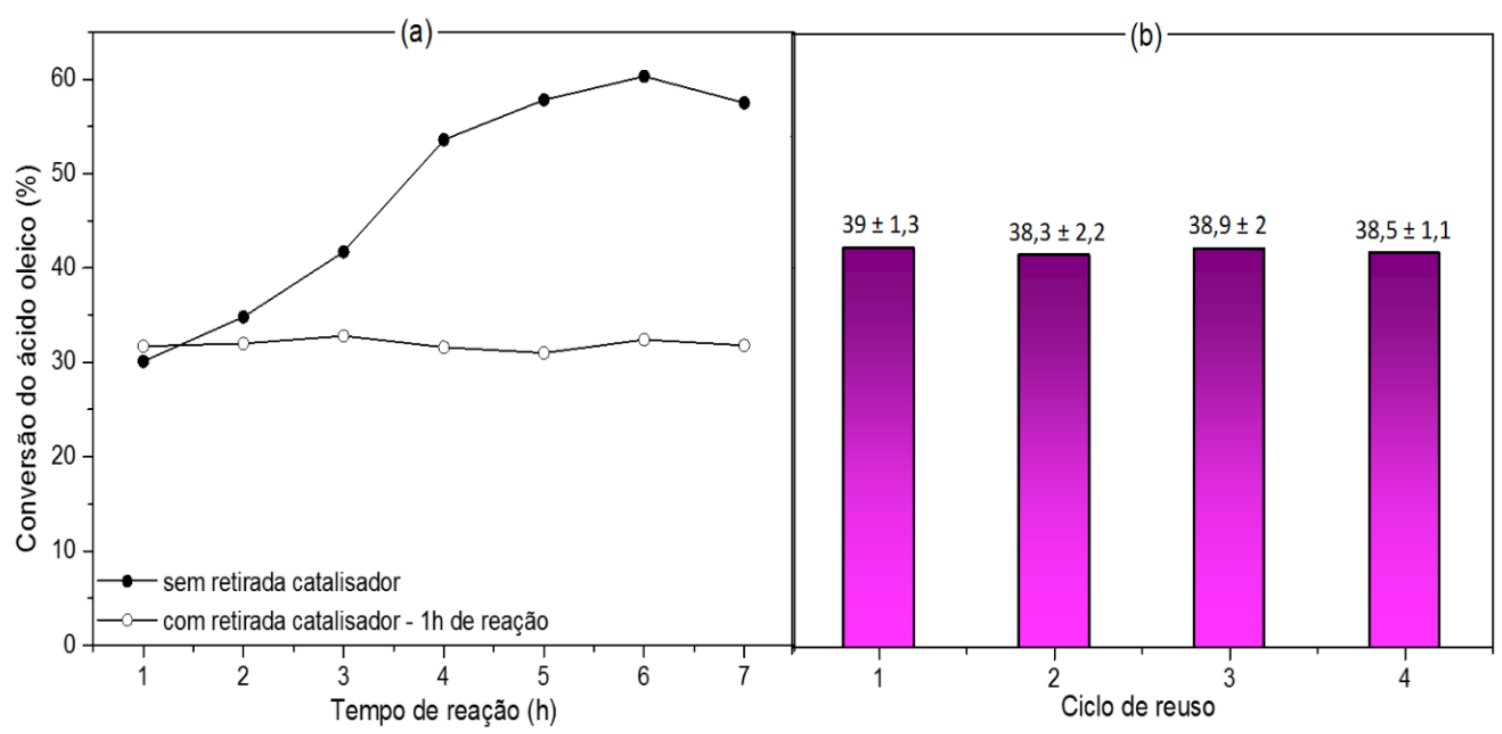

Figura 5: Teste de lixiviação (a) com retirada do catalisador após $1 \mathrm{~h}$ de reação e reutilização (b) por quatro ciclos da $\mathrm{MOF}-\mathrm{Al} / \mathrm{Zn}-\mathrm{SiO}_{2}$. Condições reacionais: razão metanol:ácido oleico $(6: 1), 5 \%$ em massa de catalisador, $150^{\circ} \mathrm{C}$ por 3 horas (reutilização). Todos os experimentos foram realizados em triplicata. Os valores de conversão (\%) foram abatidos do valor da conversão do ácido oleico na ausência do catalisador $(8,5 \pm 1,6 \%)$.

Nota-se na Figura 5 referente ao teste de lixiviação que, a atividade catalítica do material atingiu um valor máximo com 6 horas de reação. Este fato pode ser atribuído ao maior tempo de contato o que permitiu a difusão das moléculas de ácido oleico na matriz sílica até o sítio ativo da MOF-Al/Zn.

A técnica de FTIR foi utilizada a fim de se confirmar a preservação dos grupos funcionais existentes na estrutura final da MOF-Al/Zn-SiO2 após esse material ser reutilizado durante 4 ciclos catalíticos (Figura $6)$. 


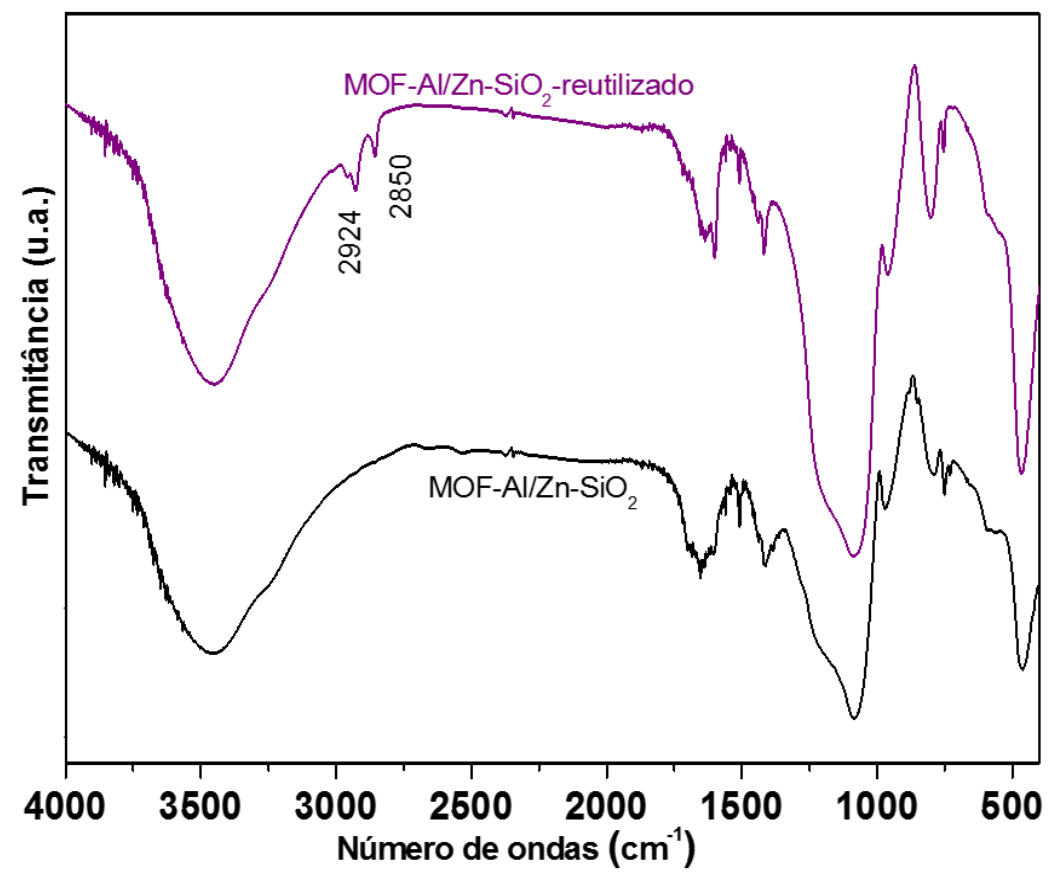

Figura 6: Espectros de absorção na região do infravermelho das amostras $\mathrm{MOF}-\mathrm{Al} / \mathrm{ZnSiO}_{2}$ antes e após o teste de reutilização por 4 ciclos.

Observa-se que os modos vibracionais referentes aos grupos silanóis ( $\mathrm{Si}-\mathrm{O}-\mathrm{Si}, \mathrm{Si}-\mathrm{OH}$ e O-Si-O) permaneceram nas regiões de 1084,969 e $459 \mathrm{~cm}^{-1}$, respectivamente. Houve um estreitamento e uma pequena variação nos comprimentos de onda para 1418,1636 e $3477 \mathrm{~cm}^{-1}$ referentes aos modos vibracionais do $\mathrm{C}=\mathrm{C}$ coordenada e não coordenado e ao grupo hidroxila, confirmando a estrutura da MOF-Al/Zn. Porém, após o catalisador ter sido reutilizado, foi observado o aparecimento de duas bandas nas regiões de 2856 e $2928 \mathrm{~cm}^{-1}$ que podem ser atribuídas à presença de componentes provenientes do meio reacional como ácido oleico ou ésteres metílicos que não foram devidamente retirados durante o processo de regeneração do catalisador por lavagem com metanol e secagem a $120^{\circ} \mathrm{C}[8,13,23]$.

\section{CONCLUSÕES}

Redes metalorgânicas (MOF) contendo $\mathrm{Al} / \mathrm{Zn}$ foram obtidas pelo método hidrotérmico, e as caracterizações físico-químicas indicaram que o método de síntese para fabricação desse material foi reprodutível e eficiente. O material MOF-Al/Zn apresentou poros maiores comparados ao material suportado na rede de sílica, porém o material MOF-Al/Zn- $\mathrm{SiO}_{2}$ apresentou maior área superficial específica e maior estabilidade térmica. O

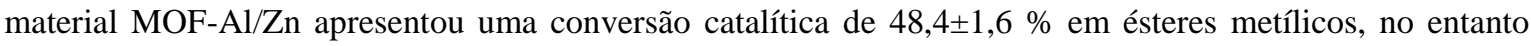
essa rede metalorgânica não obteve um bom desempenho no teste de lixiviação do material. O material

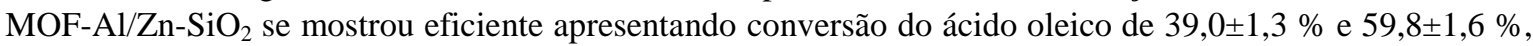
em 3 e 6 horas respectivamente. Vale ressaltar que esse material foi estável frente ao teste de lixiviação e pode ser reutilizado por 4 ciclos catalíticos sem perda significativa de atividade catalítica, indicando que a imobilização da MOF-Al/Zn na sílica é um método de síntese adequado. O material MOF-Al/Zn-SiO ${ }_{2}$ é um candidato promissor para ser empregado como catalisador heterogêneo na reação de esterificação do ácido oleico para a produção de biodiesel.

\section{AGRADECIMENTOS}

Os autores agradecem à FAPESB pelo apoio financeiro, ao CETENE - Centro de Tecnologias do Nordeste pelas análises adsorção-dessorção de nitrogênio. Ao LAPIMA e Centro de Microscopia da UESC pelas análises térmicas e MEV. LCA e FGF agradecem, respectivamente à FAPESB e a CAPES pelas bolsas de estudos. 


\section{BIBLIOGRAFIA}

[1] CHEN, H., FU, X. "Industrial technologies for bioethanol production from lignocellulosic biomass", $R e$ newable Sustainable Energy Reviews, v. 57, pp. 468-478, January 2016.

[2] DONATE, P. M. "Síntese Ambientalmente Correta a partir de Biomassa", Orbital: The Electronic Journal of Chemistry, v. 6, n. 2, April-June 2014.

[3] HAGHIGHI MOOD, S, HOSSEIN GOLFESHAN, A., TABATABAEI, M., et al., "Lignocellulosic biomass to bioethanol, a comprehensive review with a focus on pretreatment", Renewable Sustainable Energy Reviews, v. 27, pp. 77-93, July 2013.

[4] PAULOVA L., PATAKOVA P., BRANSKA B., et al., "Lignocellulosic ethanol: Technology design and its impact on process efficiency", Biotechnology Advances, v. 33, pp. 1091-1107, December 2015.

[5] GERALDES CASTANHEIRA, É., GRISOLI, R., FREIRE, F., et al., "Environmental sustainability of biodiesel in Brazil”, Energy Policy, v. 65, pp. 680-691, November 2013.

[6] ATABANI A. E., SILITONGA A. S., BADRUDDIN I. A., et al., "A comprehensive review on biodiesel as an alternative energy resource and its characteristics", Renewable Sustainable Energy Reviews, v. 16, pp. 2070-2093, February 2012.

[7] LEE, A. F., WILSON, K. "Recent developments in heterogeneous catalysis for the sustainable production of biodiesel”, Catalysis Today, v.242, March 2014.

[8] DUTTA, S. "Catalytic materials that improve selectivity of biomass conversions", RSC Advances, v. 2, pp. 12575-12593, May 2012.

[9] GUO F., FANG Z., XU C. C., et al., "Solid acid mediated hydrolysis of biomass for producing biofuels", Progress in Energy Combustion Science, v. 38, pp. 672-690, May 2012.

[10] BEOBIDE G., CASTILLO O., CEPEDA J., et al., "Metal-carboxylato-nucleobase systems: From supramolecular assemblies to 3D porous materials", Coordination Chemistry Reviews, v. 257, pp. 2716-2736, March 2013.

[11] FURUKAWA H., CORDOVA K. E., O'KEEFFE M., et al., "The Chemistry and Applications of MetalOrganic Frameworks", Science, v. 341, August 2013.

[12] DANTAS RAMOS, A. L., TANASE, S., ROTHENBER, G. G. "Redes metalorgânicas e suas aplicaçõões em catálise”, Quimíca Nova, v. 37, n. 1, pp. 123-133, August 2014.

[13] GANGU K. K., MADDILA S., MUKKAMALA S. B., et al., "A review on contemporary MetalOrganic Framework materials", Inorganica Chimica Acta, v. 446, pp. 61-74, March 2016.

[14] LI B., CHRZANOWSKI M., ZHANG Y., et al. "Applications of metal-organic frameworks featuring multi-functional sites”, Coordination Chemistry Reviews, v. 307, pp. 106-129, May 2015.

[15] NIKSERESHT A., DANIYALI A., ALI-MOHAMMADI M., et al., "Ultrasound-assisted biodiesel production by a novel composite of Fe(III)-based MOF and phosphotangestic acid as efficient and reusable catalyst”, Ultrasonics Sonochemistry, v. 37, pp. 203-207, January 2017.

[16] DANG G. H., LAM H. Q., NGUYEN A. T., et al., "Synthesis of indolizines through aldehyde - amine alkyne couplings using metal-organic framework Cu-MOF-74 as an efficient heterogeneous catalyst", Journal of Catalysis, v. 337, pp. 167-176, February 2016.

[17] SAEEDI M., FAZAELI R., ALIYAN H. "Nanostructured sodium-zeolite imidazolate framework (ZIF8) doped with potassium by sol-gel processing for biodiesel production from soybean oil", Journal Sol-Gel Science Technology, v. 77, pp. 404-415, 2016.

[18] HASAN Z., JUN J. W., JHUNG S. H. "Sulfonic acid-functionalized MIL-101(Cr): An efficient catalyst for esterification of oleic acid and vapor-phase dehydration of butanol", Chemical Engineering Journal, v. 278 , pp. 265-271, September 2014.

[19] KARMAKAR A., GUEDES DA SILVA M. F. C., POMBEIRO A. J. L. "Zinc metal-organic frameworks: efficient catalysts for the diastereoselective Henry reaction and transesterification", Dalton Transicitions, v. 43, pp. 7795-7810, February 2014.

[20] CORMA A., GARCIA H., LLABRES, I. X. F. X. "Engineering metal organic frameworks for heterogeneous catalysis”, Chemical Reviews, v. 110, pp. 4606-4655, 2010.

[21] YANG L., RUESS G. L., CARREON M. A. "Cu, Al and Ga based metal organic framework catalysts for the decarboxylation of oleic acid”, Catalysis, Science and Technology, v. 5, pp. 2777-2782, February 2015 . 
[22] AKHTAR F., ANDERSSON L., OGUNWUMI S., et al., "Structuring adsorbents and catalysts by processing of porous powders", Journal of the Europen Ceramic Society, v. 34, pp. 1643-1666, January 2014.

[23] CHUGHTAI A. H., AHMAD N., YOUNUS H. A., et al., "Metal-organic frameworks: versatile heterogeneous catalysts for efficient catalytic organic transformations", Chemical Society Reviews, v. 44, pp. 68046849, May 2015.

[24] VLASOVA E. A., SHALUNOVA N.K., MAKAROVA A. S., et al., "Metal-organic frameworks based on terephthalic acid: Sorbents of organic dyes", Russian Journal of Applied Chemistry, v. 87, n. 8, pp. 10651069, May 2015.

[25] ARNOLD U., CRUZ R. S., MANDELLI, D., et al., "Activity, selectivity and stability of metallosilicates containing molybdenum for the epoxidation of alkenes", Journal of Molecular Catalysis A: Chemical, v. 165, pp. 149-158, 2001.

[26] SÁNCHEZ-SÁNCHEZ, M., GETACHEW, N., DÍAZ, K., et al., "Synthesis of metal-organic frameworks in water at room temperature: salts as linker sources", Green Chemistry, v. 17, pp. 1500-1509, 2015.

[27] VLASOVA E. A., NAIDENKO E. V., KUDRIK E. V., et al., "Efficient synthesis of aluminum- and zinc-containing metal-organic frameworks", Inorganic Materials, v. 51, pp. 236-240, 2015. 